\title{
A ureteral mass presenting as deep vein thrombosis: a very rare presentation
}

\begin{abstract}
Venous Thromboembolism is a dreaded condition. Many conditions and systemic diseases are known to predispose to its occurrence including malignancy, pancreatitis, burns, clotting disorders and direct compression. All of these have been known to affect one or more factors in Virchows Triad proposed roughly a century ago. ${ }^{1}$ It consists of Hypercoagulability, Vascular Endothelial Dysfunction, Stasis. Stasis, one of the factors in Virchows triad has been studied to a very small extent and it is this feature that is mostly responsible for the development of Deep Vein Thrombosis (DVT) due to direct compression. Iliofemoral thrombosis in malignancy patients can be caused due to vein compression by pelvic malignancy and usually presents as unilateral lower limb swelling. In patients with sudden onset of unilateral lower limb swelling without any perceived or diagnosed medical condition, detailed evaluation may lead to early identification, appropriate management and possibly cure.
\end{abstract}

We present a case of acute unilateral ilio-femoral DVT caused by external compression by a mid-ureteric mass.

Keywords: deep vein thrombosis, ileofemoral thrombosis, ureteric malignancy, upper tract urothelial cancers
Volume 6 Issue 3 - 2018

\author{
Dushiant Sharma, Umesh Sharma, Sumit \\ Gehlawat
}

Department of Urology, RML Hospital \& PGIMER, Delhi, India

Correspondence: Dushiant Sharma, Department of Urology Dr RML Hospital \& PGIMER, Baba Khadak Singh Marg, New Delhi, I I000I,Tel 0I I-2340-4323,Email dushiant@gmail.com

Received: November 15, 2018 | Published: June 22, 2018
Abbreviations: DVT, Deep Vein Thrombosis; VTE, Venous Thromboembolism

\section{Case presentation}

A 45 year old post-menopausal female presented to her primary physician with complaints of left foot swelling which gradually progressed to involve the entire left lower limb. She did not give any antecedent history of trauma, ulceration on lower limb, haematuria, lower urinary tract symptoms, bowel complaints, vaginal bleeding, loss of weight, loss of appetite, previous history of DVT. There was no history of previous surgeries and co-existing morbidities. Her left lower limb was reddish in color, tense and tender to touch. Pervaginal examination revealed induration with in the left fornix. Rest of the general and systemic examination, were within normal limits. A venous Doppler revealed a partial thrombus of the Left Proximal external iliac vein and Left Common Iliac vein. Thrombophillic screen (PT-INR, aPTT, Protein C, Protein S, D, and Factor V Leiden Mutation) was within normal limits. Patient was started on Warfarin and put on total bed rest. Due to absence of any predisposing factors, further evaluation was considered and an ultrasound of the abdomen was done. It revealed a grossly dilated left renal pelvis with thinned out parenchyma \& a $1.3 * 1.1 \mathrm{~cm}$ lesion near the left vesicoureteric junction. Contrast-Enhanced CT of Abdomen \& Pelvis Figure 1 was done which shows poorly functioning left kidney with gross hydronephrosis \& thinned out parenchyma, large $6.6 * 4.5 \mathrm{~cm}$ heterogeneously enhancing solid cystic mass in the left ureter at the level of the iliac vessel crossing and $3.5^{*} 2.1 \mathrm{~cm}$ heterogeneously enhancing solid cystic lesion in left distal ureter extending up to VUJ. Her hemoglobin, serum creatinine, liver function tests, urinalysis were within normal limits. Urine for malignant cytology was negative. Cystoscopy showed a bulge just lateral to the left ureteric orifice and no other visible mass. The left ureteric orifice could not be cannulated. Considering the diagnosis of a ureteric mass, a Left
Nephrouretectomy with bladder cuff excision was planned. Warfarin was stopped 5 days before surgery and patient was put on bridging therapy with low molecular weight heparin. A cardiology consult was taken and it was decided to insert an IVC filter 24 hours before surgery as prophylaxis to decrease risk of intra-operative and postoperative pulmonary embolism. Intra-operatively DVT pump was not applied. A Radical Nephroureterectomy along with Hysterectomy and Left Salpingo-oophorectomy (Figure 2) (Figure 3) was done with gynecological assistance in view of local invasion of cervix. Mid ureteric mass was dissected off the iliac vessels with CTVS assistance and final specimen consisted of the Left kidney, ureter, Uterus, Left Ovary along with Lymph Node dissection. Total intra-operative blood loss was $600 \mathrm{ml}$. Post-operatively patient was kept in the ICU before shifting to the ward. Warfarin was re-started on day 3. Patient had a relatively uneventful post-operative period.

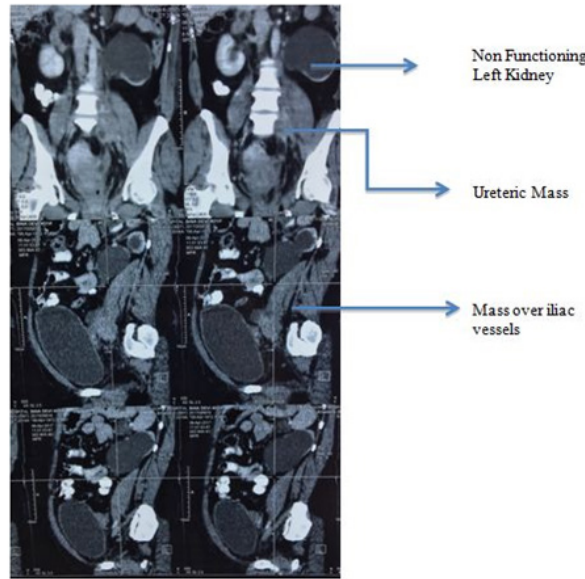

Figure I Pre-op CECT Abdomen + Pelvis( Upper half - Coronal Section, Lower half- Sagittal section. 


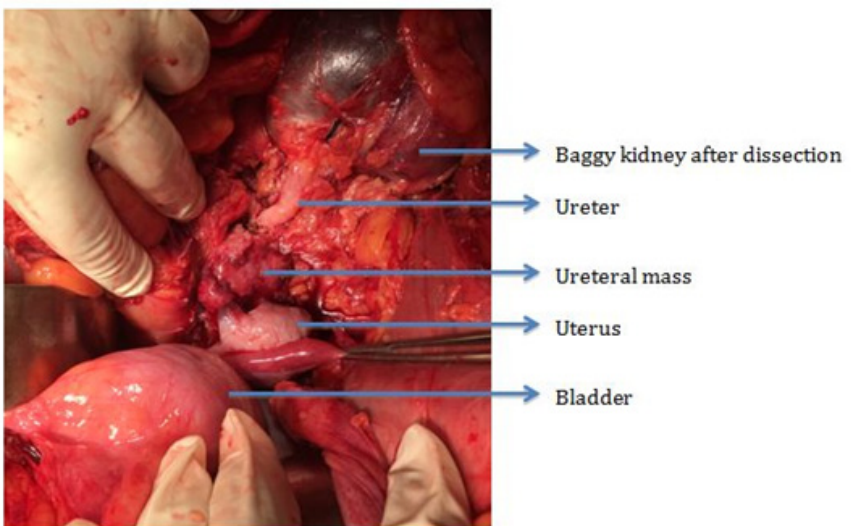

Figure 2 Baggy Kidney with uretric mass. Bladder retracted in the lower part of the photo.

HPE revealed a high grade Urothelial Carcinoma of the Ureter with focal squamous differentiation with invasion into cervix and uterus Lymph nodes were free of tumor. Final stage was pT4 N0 Mx. Patient was given 3 cycles of Adjuvant Chemotherapy till time of abstract submission (Gemcitabine+ Cisplatin) and was doing well at final follow up with resolution of DVT. Follow up CECT abdomen+ Pelvis is planned but the patient is yet to follow up despite repeated reminders

\section{Discussion}

Upper tract urothelial carcinomas are a rare entity comprising only $5-10 \%$ of all urothelial tumors but recent literature reveals an increasing incidence. ${ }^{2}$ Ureteral tumors occur more commonly in the lower than in the upper ureter. Overall, about $70 \%$ of ureteral tumors occur in the distal ureter, $25 \%$ in the mid-ureter, and 5\% in the proximal ureter. ${ }^{3,4}$ The most common clinical feature of urothelial carcinoma of the ureter is haematuria, either gross or microscopic, present in roughly 56-98\% cases,${ }^{5-7}$ followed by flank pain in $30 \%$ cases. Urothelial carcinoma of ureter presenting as unilateral lower limb swelling secondary to DVT is very rare. Venous thromboembolism (VTE) risk factors have been classified into- Modifiable, Non- modifiable and Temporary. ${ }^{8}$ Modifiable risk factors include obesity. Non modifiable risk factors include genetic and familial disorders resulting in a hypercoagulable state. Temporary risk factors include hospitalization, malignancy, long distance travel, prolonged immobilization, hormonal therapy for various diseases. But most of the VTE is still labeled as 'Idiopathic' without any known antecedent cause.

Malignant compression of iliofemoral veins in the pelvis by malignancies is one such case in which patients maybe mistakenly labeled as having idiopathic VTE with potentially disastrous outcomes. Ureteric masses causing direct iliofemoral venous compression with resultant DVT and unilateral lower limb swelling have seldom been reported. Most of the reported cases of Iliofemoral venous compression with resultant DVT have been due to compression by enlarged lymph nodes secondary to Urothelial or gynecological malignancies. Liao et al., ${ }^{9}$ analyzed 893 cancer patients over a 10 year period.

The inclusion criteria for their study were:

1. presence of unilateral lower limb swelling,

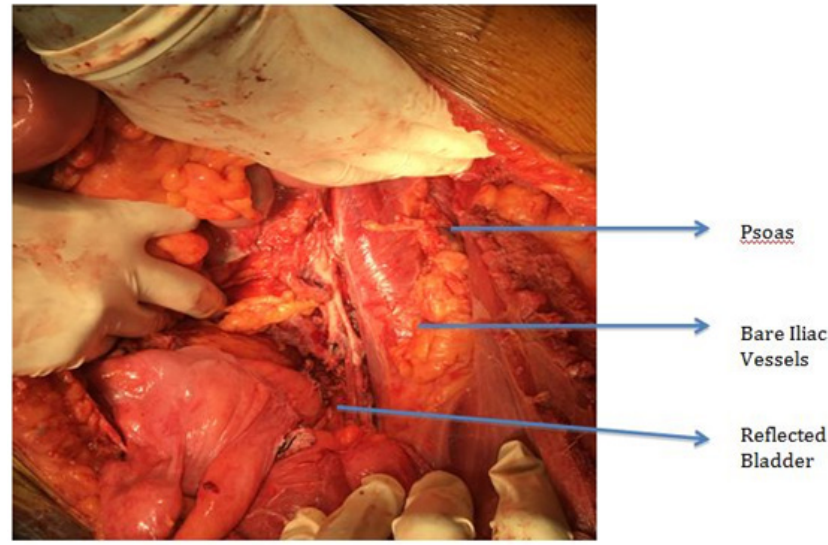

Figure 3 Tumor bed with exposed iliac vessels after removal of Left kidney, Ureter, Left ovary.

2. a CT showing malignancy causing external compression on iliac or femoral vein and

3. a duplex ultrasound revealing vein thrombosis or venous flow insufficiency over a femoral vein or saphenous vein.

Patients with bilateral lower limb edema suggestive of IVC involvement were excluded. $63(8 \%)$ of these patients were diagnosed with iliofemoral venous obstruction. 21 of these 63 patients developed iliofemoral venous thrombosis. Most of the compression was by lymph nodal metastasis secondary to urothelial cancers (46). Bladder was the most common primary site (29) followed by ureter $(n=8)$, renal pelvis $(n=5)$, prostate $(n=2)$, or penis $(n=2)$ but none of these were due to direct malignant compression by Ureteric Mass. Other causes of iliofemoral venous compression included the most commonly described syndrome called the Iliac Vein Compression Syndrome or the May-Thurner Syndrome involving compression of Left Iliac Vein by Right Iliac Artery. ${ }^{10}$

Many other rare causes of iliofemoral compression and thrombosis have been reported in literature including uterine fibroids, ${ }^{11}$ psoas abscess, ${ }^{12}$ retroperitoneal fibrosis, ${ }^{13}$ penile prosthesis reservoir, ${ }^{14}$ synovial cyst of hip. ${ }^{15}$ Other urological entities have also been reported to cause iliofemoral venous thrombosis. Alliota et al., ${ }^{16}$ reported a giant hydronephrosis as a cause of iliofemoral thrombosis. Bladder diverticulae have also been known to cause iliofemoral thrombosis according to various reports. ${ }^{17-19}$ Through our case report, we would like to emphasize the need for thorough evaluation of patients presenting with unilateral lower limb swelling due to DVT without any predisposing factors. A simple ultrasound of the abdomen or contrast enhanced CT might shed light on a sinister underlying malignancy. Proper diagnosis will guide therapy and will lead to early management of malignancy resulting in better prognosis.

\section{Acknowledgements}

We would like the thank the patient for giving us permission to publish the detailes of her case. We would like to thank the Department of Pathology for providing with the Histopathological diagnosis which helped in further post-operative management of our patient. 


\section{Conflict of interest}

Author declares there is no conflict of interest in publishing the article.

\section{References}

1. Bagot $\mathrm{CN}$, Arya R. Virchow and his triad: a question of attribution. $B r J$ Haematol. 2008;143(2):180-90.

2. Siegel R, Naishadham D, Jemal A. Cancer statistics, 2013. CA Cancer J Clin. 2013;63(1):11-30.

3. Anderstrom C, Johansson SL, Pettersson S, et al. Carcinoma of the ureter: a clinicopathologic study of 49 cases. J Urol. 1989;142(2):280-3.

4. Messing EM, Catalona W. Urothelial tumors of the urinary tract. $7^{\text {th }} \mathrm{ed}$. Walsh PC, Retik AD, Vaughan ED, et al, editors. Campbell's urology. Philadelphia: Saunders; 1998. p. 2327-410.

5. Murphy DM, Zincke H, Furlow WL. Management of high grade transitional cell cancer of the upper urinary tract. J Urol. 1981;125:25-9.

6. Guinan P, Vogelzang NJ, Randazzo R, et al. Renal pelvic cancer: a review of 611 patients treated in Illinois 1975-1985. Urology. 1992;40(5):393-9.

7. Raabe NK, Fossa SD, Bjerkehagen B. Carcinoma of the renal pelvis. Scand J Urol Nephrol. 1992;26(4):357-361.

8. Cushman M. Epidemiology and risk factors for venous thrombosis. Semin Hematol. 2007;44(2):62-9.

9. Liao TY, Hsu HC, Wen MS, et al. Iliofemoral Venous Thrombosis Mainly Related to Iliofemoral Venous Obstruction by External Tumor Compression in Cancer Patients. Case Reports in Oncology. 2016;9(3):760-71.
10. May R, Thurner J. The cause of the predominantly sinistral occurrence of thrombosis of the pelvic veins. Angiology. 1957;8(5):419-27.

11. Khilanani R, Dandolu V. Extensive iliac vein thrombosis as a rare complication of a uterine leiomyoma: a case report. J Reprod Med. 2007;52(6):537-8.

12. Arai Y, Kawakami T, Soga H, et al. Psoas abscess associated with iliac vein thrombosis and piriformis and gluteal abscesses. Int $J$ Urol. 1999;6(5):257-9.

13. Rhee RY, Gloviczki P, Luthra HS, et al. Iliocaval complications of retroperitoneal fibrosis. Am J Surg. 1994;168(2):179-83.

14. Da Justa DG, Bianco FJ, Ogle A, et al. Deep venous thrombosis due to compression of external iliac vein by the penile prosthesis reservoir. Urology. 2003;61(2):462.

15. Sugiura M, Komiyama T, Akagi D, et al. Compression of the iliac vein by a synovial cyst. Ann Vasc Surg. 2004;18(3):369-71.

16. Aliotta PJ, Lacey SR, Allen JE, et al. Giant hydronephrosis presenting as unilateral iliofemoral vein thrombosis. J Urol. 1988;139(5):1035-6.

17. Murchison C. Obstruction and inflammation of the femoral vein resulting from the pressure of a distended sacculus of the urinary bladder. Trans Pathol Soc London. 1863;14:133-7.

18. Jepson PM, Nickson KL, Silber I. Giant vesical diverticula with thrombophlebitis. Urology. 1973;1:606-8.

19. Gupta V, Shaik I, Abbas J, et al. Iliofemoral venous thrombosis from external compression by a vesical diverticulum. J Vasc Surg. 2010;52(6):1671-3. 